\title{
1 Astroglia in lateral habenula is essential for antidepressant efficacy of light
}

3 Sarah Delcourte ${ }^{1 \S}$, Adeline Etievant ${ }^{2}$, Renaud Rovera ${ }^{1}$, Damien Mor $^{2}$, Howard M Cooper ${ }^{1}$, Hiep 4 D. Le ${ }^{3}$, April E. Williams ${ }^{4}$, Satchidananda Panda ${ }^{3}$, Rihab Azmani ${ }^{1}$, Olivier Raineteau ${ }^{1}$, Christine 5 Coutanson ${ }^{1}$, Ouria Dkhissi-Benyahya ${ }^{1 \S}$, Nasser Haddjeri ${ }^{1}{ }^{*}$

6 1. Univ Lyon, Université Claude Bernard Lyon 1, Inserm, Stem Cell and Brain Research Institute 7 U1208, 69500 Bron, France

8 2. Integrative and Clinical Neurosciences EA481, University of Bourgogne Franche-Comté, 9 Besançon, France.

10 3. Regulatory Biology Laboratory, Salk Institute for Biological Studies, 10010, North Torrey Pines 11 Road, La Jolla, CA 92037, USA

12 4. Razavi Newman Integrative Genomics and Bioinformatics Core, The Salk Institute for Biological 13 Studies, La Jolla, California equally

17

18 The authors have declared that no conflict of interest exists.

19

$20 \quad{ }^{*}$ Corresponding author: Nasser Haddjeri, Ph.D.

21 INSERM U1208, Stem Cell and Brain Research Institute,

22 Université Lyon I, 18, avenue du Doyen Jean Lépine

2369500 Bron France

24 Tel: (33) 4-72-91-34-78

25 Email: nasser.haddjeri@inserm.fr 


\section{Abstract}

29 Successful antidepressant (AD) treatments are still difficult to achieve. Recently, bright light

30 stimulation (BLS) was shown effective in non-seasonal depression but its mode of action remains

31 elusive. We demonstrate here, using a new mouse model of depression resistant to ADs including

32 ketamine, that chemogenetic activation of lateral habenula ( $\mathrm{LHb}$ ) astroglia prevented the

33 potentiating effect of BLS on the AD response. Additionally, the beneficial action of BLS was

34 associated with upregulation of a specific part of the prefrontal cortex opioid system. These results

35 show that improved behavioral outcome produced by BLS requires habenular astroglia and

36 endogenous opioids as crucial buffer systems.

\section{Introduction}

42 Although major depression (MD) is the most common psychiatric disorder worldwide, fully effective

43 treatments to cure MD are still awaiting. One of the safer, low-cost and non-invasive therapeutic

44 candidates is bright light stimulation (BLS). Light has a critical broad role in health, acting directly

45 or through the circadian system to modulate brain structures involved in sleep regulation, mood

46 and cognition (LeGates et al., 2014). Hence, aberrant light cycles produce depressive behaviors

47 and impair cognition in animals and in humans (LeGates et al., 2014; Fernandez et al., 2018).

48 Moreover, the prevalence of MD is increased in night-workers, in whom both light exposure and

49 circadian rhythmicity are altered. Inversely, depressed patients frequently display disturbed

50 circadian rhythms and sleep/wake cycles (McClung, 2013). Since 3 decades, BLS is the first-line

51 choice for the treatment of seasonal depression (Wirz-Justice et al., 2004). Surprisingly, BLS

52 treatment has also proven to be effective in non-seasonal depression and even more efficient than

53 the classical AD Prozac (Lam et al., 2016). Paradoxically, the mechanisms involved in the 
54 therapeutic effect of light are still unknown. The main objective of this study was to assess the

55 involvement of the circadian, habenular and 5-HT systems, three important interactive brain

56 networks regulating mental health (Metzger et al., 2017) and playing a key role in mood regulation

57 by light (LeGates et al., 2014; Fernandez et al., 2018; Huang et al., 2019).

\section{Results \& Discussion}

60 The current 'gold-standard' behavioral test to examine the efficacy of potential AD agents is the

61 forced swimming test (FST) in which ADs are well known to decrease immobility and increase

62 active coping behavior (swimming and climbing) (Porsolt et al., 1997). To provide a reliable mice

63 model of depression, we recently proposed that the timing of stress and testing is a critical factor

64 for 5 days Repeated Forced Swim Stress (5d-RFSS) model (Sun et al., 2015; Delcourte et al.,

65 2017). In the present study, 6-week old C57BL/6J mice socially isolated were forced to swim during

66 the dark/active phase on 5 successive days for 10 minutes at zeitgeber time 14 (ZT14) and were

67 then tested every week. A stress at ZT14 was extremely effective to induce a long-lasting (up to 8

68 weeks) depressive-like behavior (Fig. 1A). Therefore, mice exposed to the 5d-RFSS expressed a

69 reduced sucrose preference indicating the presence of an anhedonia phenotype (Fig. 1B). In

70 addition, the stressed mice exhibited higher anxiety and fear levels, measured as reduced time

71 spent in the open arms in the elevated plus maze, as well as an increased latency to feed in the

72 novelty suppressed feeding test (Fig. 1C, 1D). Treatment with the selective 5-HT reuptake inhibitor

73 AD escitalopram, or the NMDA receptor antagonist ketamine produced an AD-like behavior in naïve

74 mice (Fig $1 \mathrm{~F}-\mathrm{H})$. In contrast, these treatments were ineffective to reverse the behavioral despair in

75 5d-RFSS mice (Fig. 1E-G), thus revealing $5 d$-RFSS as a new pharmaco-resistant model of

76 depression. Remarkably, among all antidepressants tested in the current study, only a sub-chronic

77 treatment with the mixed opioid agent buprenorphine (a potent mu opioid receptor partial agonist 
78 and kappa opioid receptor antagonist, Falcon et al., 2016) showed an AD-like response in 5d-

79 RFSS mice (Fig S1).

80 Using the FST as a main AD readout, we then assessed the effect of BLS alone or in combination

81 with $A D$ agents on this refractory model. BLS failed to induce an AD response given alone (Fig.

82 2B). While a combination of sub-effective doses of ketamine and the muscarinic receptor antagonist

83 scopolamine produced an AD-like behavior in naïve mice (Petryshen et al., 2016; Fig.2A), the

84 latter antagonists did not reverse the behavioral despair induced by the 5d-RFSS (Fig. 2C).

85 Importantly, we found that BLS added to a combination of ketamine and scopolamine, reduced the

86 immobility time observed in the FST, indicating that BLS potentiated the AD response of the latter

$87 \quad A D$ treatment (Fig. 2C).

88 Several lines of evidence significantly link the effects of light to 5-HT regulation of mood (Gonzalez

89 \& Aston-Jones, 2008; Defrancesco et al., 2013). Interestingly, the AD-like effect of ketamine, but

90 not scopolamine, was abolished by $5-\mathrm{HT}$ depletion produced by the tryptophan hydroxylase

91 inhibitor, para-chlorophenylalanine (PCPA) (Giglucci et al., 2013; Palucha-Poniewiera et al.,

92 2017). Accordingly, our results showed that the potentiating effect of BLS on the action of the

93 ketamine/scopolamine combination was prevented by PCPA pre-treatment, revealing a permissive

94 role of the 5-HT system in the AD behavioral phenotype (Fig. 2D).

$95 \mathrm{LHb}$ has emerged as a key structure interface between light effects and 5-HT regulation of mood.

$96 \mathrm{LHb}$, which receives projections from the intrinsically photosensitive retinal ganglion cells (Hattar

97 et al., 2006), is anatomically and functionally connected to the raphe 5-HT nuclei (Pasquier et al.,

98 1976). While it was firstly shown that pseudo-depressed mice display increased activity of LHb

99 neurons (Lecca et al., 2016), Yang et al (2018) recently reported in the LHb that coordinated

100 activity of NMDA receptors and T-type voltage-sensitive $\mathrm{Ca2}+$ channels cause neuron firing to

101 occur in a pattern of rapid bursts and that such aberrant firing leads to depressive-like symptoms.

102 Additionally, Cui et al. (2018) revealed the involvement of LHb astroglia in the increases of burst

103 firing mode and depressive-like phenotype. Hence, we sought to determine whether selective

104 chemogenetic activation of transduced astroglia in LHb, could counteract the potentiating AD action 
105 of BLS in 5d-RFSS mice. Injection of the GFAP-Gq-DREADD virus in the LHb showed fields of 106 mCherry-positive cells, that consistently express the GFAP astroglia marker and lack the NeuN

107 neuronal marker (Fig. 2G-H). Remarkably, clozapine-n-oxide (CNO) administration, by producing 108 a selective and robust activation of astrocytes in mice expressing Gq-DREADD in LHb astrocytes, 109 significantly prevented the potentiating effect of BLS on the action of the ketamine/scopolamine 110 combination (Fig. 2E). This result reveals a crucial involvement of $\mathrm{LHb}$ astroglia as well as a 111 permissive role of $5-\mathrm{HT}$ and opioid systems in the beneficial action of BLS in the 5d-RFSS 112 pharmaco-resistant model. Recently, the antidepressant effects of ketamine were shown to be 113 attenuated by opioid receptor blockade in both human and rodent (Williams et al., 2019; Klein et 114 al., 2020). Importantly, the reducing action of ketamine on LHb cellular hyperactivity is also 115 prevented by opioid receptor blockade (Klein et al., 2020). To further characterize both 5d-RFSS 116 model and BLS action, we performed RNA-seq in the PFC, a key brain region involved in both MD 117 (Krishnan \& Nestler, 2008) and antidepressant response (including ketamine) (Gerhard et al., 118 2019). Remarkably and in full agreement with the AD response of buprenorphine, the efficient co119 treatment with light modified opioid system gene expression in PFC with a peculiar enhancement 120 of Opioid Receptor Mu-type 1 (OPRM1), Opioid Receptor Kappa-type 1 (OPRK1), Proenkephalin 121 (PENK) and Prodynorphin (PDYN) gene expressions, thus demonstrating for the first time that BLS 122 potentiated the effect of pharmacological treatments by mechanisms involving the expression of 123 opioid-related genes in PFC (Fig S2).

124 As a translational outcome, the current study will certainly have far-reaching impact on our 125 understanding of the mechanisms involved in light effects on depression to ultimately optimize 126 therapeutic strategies in drug-resistant or refractory depressed patients (including partialresponders to ketamine) and proposes BLS as a beneficial strategy against the possible deleterious 
128 effect induced by social isolation such as e.g. the current one required against the Covid-19's

129 pandemic.

\section{Methods}

\section{Animals}

132 All animal procedures were in strict accordance with current national and international regulations

133 on animal care, housing, breeding, and experimentation and were approved by the regional ethics

134 committee CELYNE (C2EA42-13-02-0402-005). All efforts were made to minimize suffering. 6

135 week-old C57BL/6J male mice (25-30 g on arrival; Charles River Laboratories) were individually

136 housed in a temperature- and humidity-controlled environment with a $12 \mathrm{~h}$ dark/12 light (12L/12D)

137 cycle. Mice received food and water ad libitum and were allowed 2 weeks to acclimate before the

138 experiments.

\section{5 days Repeated Forced Swim Stress (5d-RFSS)}

140 Two hours after lights off (ZT14), mice were placed in a tank filled with water $\left(25^{\circ} \mathrm{C}\right)$ and forced to

141 swim 10 minutes daily for 5 consecutive days. The immobility time of the first 4 minutes of the stress

142 was analyzed. Then, every week, a forced swim test (FST) was realized to follow the evolution of

143 the immobility time.

\section{Sucrose preference test}

145 Mice were habituated to 2 bottles of $1 \%$ sucrose during $24 \mathrm{~h}$. Then, they were given the choice to

146 drink from 2 bottles (1\% sucrose solution and tap water bottle) during $72 \mathrm{~h}$. The positions of the

147 bottles in the cage were switched every $24 \mathrm{~h}$ to avoid possible side-preference effects.

148 Elevated plus maze

149 Animals were placed in the room half an hour before the beginning of the test. Experiments were 150 realized during the light phase of the 12L/12D cycle. The apparatus consisted of 2 Plexiglas open 151 arms $(6 \times 96.5 \mathrm{~cm})$, and 2 closed arms $(6 \times 95.5 \mathrm{~cm})$, surrounded by $15 \mathrm{~cm}$ black high walls elevated $15250 \mathrm{~cm}$ above the floor. At the beginning of the test, the mouse was placed in the central platform of 
153 the maze, facing an open arm. Each session lasted 6 min and was video recorded to analyze the

154 time spent in the open arms.

\section{$155 \quad$ Novelty suppressed feeding test}

156 The latency to begin eating a pellet of food in a frightening environment is considered as a measure

157 of anxio/depressive-like behavior. Animals were food-deprived for $24 \mathrm{~h}$ prior to behavioral testing.

158 Mice were individually placed in a brightly illuminated plastic box $(50 \mathrm{~cm} \times 40 \mathrm{~cm} \times 20 \mathrm{~cm})$ covered

159 with wooden bedding. A single food pellet (regular chow) was placed on a white paper platform

160 positioned in the center of the arena. The latency to eat (defined as the mouse sitting on its

161 haunches and biting the pellet with the use of forepaws) was timed. The NSF test was carried out

162 during a 5 min period.

\section{Drugs}

164 Escitalopram was supplied by $\mathrm{H}$. Lundbeck A/S. Ketamine hydrochloride was purchased from

165 Sigma-Aldrich. Scopolamine hydrobromide and Clozapine-N-oxide (CNO) were purchased from

166 Tocris Biosciences, Buprenorphine (Subutex) was kindly provided by Bruno Guiard (CRCA,

167 Toulouse). All drugs were dissolved in a 0,9\% saline solution. Escitalopram sub-chronic treatment

168 was administered at $10 \mathrm{mg} / \mathrm{kg}$, i.p during 5 consecutive days following the $5 \mathrm{~d}-\mathrm{RFSS}$, or during the

169 five preceding the FST for the naïve group. Buprenorphine $(0,3 \mathrm{mg} / \mathrm{kg}$, i.p.) was administered every

170 two days five times. Ketamine hydrochloride was administered acutely at $10 \mathrm{mg} / \mathrm{kg}$ alone or in

171 combination with scopolamine $(0,1 \mathrm{mg} / \mathrm{kg}$, i.p), at $3 \mathrm{mg} / \mathrm{kg}$, i.p. Ketamine and scopolamine were 
172 administered 30 minutes before the forced swimming test. Clozapine-N-oxide (CNO) was dissolved

173 in saline and administered at $1 \mathrm{mg} / \mathrm{kg}$, i.p. 30 minutes before the FST.

\section{$174 \quad$ Light stimulation protocols}

175 At the end of the 5d-RFSS, mice were exposed every day to a bright white light (1000 lux; LED

176 bulb) for one hour at ZT11. The effect of light exposure on depressive-like behavior was analyzed

177 every week during 4 weeks post 5d-RFSS.

$178 \quad$ PCPA treatment

179 Three days before the 4-week's FST, animals were injected each day with $150 \mathrm{mg} / \mathrm{kg}$ of 4-Chloro-

180 DL-phenylalanine (PCPA, Sigma Aldrich), the last day of injection was $24 \mathrm{~h}$ before the last FST.

181 Surgery

182 Mice were anesthetized with Chloral Hydrate (30m/kg, i.p.; SIGMA) and Xylazine (12 mg/kg i.p.;

183 Bayer). There were implanted with cannulas for the virus microinjections. The vector, containing

184 the virus, was dissolved in PBS $+\mathrm{MgCl} 2+\mathrm{KCl}$ at a concentration of $6,0.1012 \mathrm{vg} / \mathrm{mL} .0,2 \mu \mathrm{l}$ of

185 ssAAV-5/2-hGFAP-hM3D(Gq)-mCherry-WPRE-hGHp(A) (GFAP-Gq-DREADD; Viral vector

186 Facility- Zurich University) was infused bilaterally into the LHb (AP + 4.24mm; ML士 0.48; DV +

$1874.2 \mathrm{~mm}$ from bregma with a $45^{\circ}$ angle) using 33 gauge injectors at rate of $0.05 \mu \mathrm{l} / \mathrm{min}$.

\section{Immunohistochemical Staining}

189 Animals were given pentobarbital (50 mg/kg, i.p.) and trans-cardiac perfused with $4 \%$ 190 paraformaldehyde (PFA). Brains were removed and post-fixed in $4 \%$ PFA at $4^{\circ} \mathrm{C}$ for an additional $19124 \mathrm{~h}$, rinsed in PBS (phosphate-buffered saline, $\mathrm{pH} 7.4$ ), and cryo-protected in $30 \%$ sucrose in 0.1

$192 \mathrm{M}$ PBS for an additional $48 \mathrm{~h}$ at $4^{\circ} \mathrm{C}$. Brains were harvested and sliced at $30 \mu \mathrm{m}$, and incubated for $19324 \mathrm{~h}$ with anti-GFAP (Mouse, 1:500, Sigma), anti-NeuN (Guinea Pig, 1:500, Synaptic systems) and 194 anti RFP (Rabbit, 1:1000, MBL) antibodies in PBS $+5 \%$ normal goat serum. RFP signal was 195 amplified using a biotinylated anti-rabbit secondary antibody (Goat, 1:200, Vector Biosystem) and 196 streptavidin-DTAF complex (1:250, Jackson Labs Technologies) for 30 min. GFAP, NeuN, and 197 RFP stainings were visualized using respectively Alexa Fluor 488, 647 (Donkey 1:500 Jackson 
198 Immunoresearch) and Streptavidin-Cy3 (1:500, Jackson Immunoresearch). Images were obtained

199 with a Leica SP5 confocal (Leica Microsystems). Z-series images were taken at $2 \mu \mathrm{m}$ intervals.

\section{RNA extraction}

201 Two days after the last forced swim test, mice were euthanized. Both sides of the medial prefrontal

202 cortices were dissected on ice and stored at $-80^{\circ} \mathrm{C}$. Total RNAs were extracted using Trizol reagent

203 (Invitrogen) according to manufacturer's instructions.

204 Libraries preparation and high-throughput sequencing Libraries were prepared using

205 Illumina's TruSeq Stranded mRNA HT kit according to manufacturer's instructions. In brief, total

206 RNA starting with 700ng was poly-A selected, fragmented by metal-ion hydrolysis and then 207 converted to cDNA using SuperScript II. The cDNA was then end-repaired, adenylated and ligated 208 with Illumina sequencing adapters. Finally, the libraries were enriched by 15 cycles of PCR 209 amplification. Libraries were pooled and sequenced using an Illumina HiSeq 2500 with 50-bp 210 single-read chemistry.

\section{Transcriptome data analysis}

212 Whole-transcriptome profiling of prefrontal cortices was performed by RNASeq. Sequenced reads 213 were mapped to the reference mouse genome using (org.Mm.eg.db ,

214 http://bioconductor.org/packages/release/data/annotation/html/org.Mm.eg.db.html). Read counts 215 were generated using Homer and normalized counts were used in DESeq2 (Bioconductor , 216 http://bioconductor.org/packages/release/bioc/html/DESeq2.html) to measure differential 217 expression. The RNASeq raw data are available at GEO under accession number GSE143820.

218 The candidate genes were screened from a comparison of control and stressed mice, as well as of 219 stressed mice treated with light, scopolamine and ketamine (i.e. KScL mice) as follows: first, the 220 normalized counts were cut-off at 25 in at least one of the samples, then the gene lists were 221 generated based on adjusted $p$-value $<0.01$. Lists of genes were submitted to DAVID 222 (https://david.ncifcrf.gov) for functional enrichment analysis. 


\section{Author Contributions}

225 S.D., A.E., O.D-B and N.H. designed research; S.D. and A.E. performed research; R.R., D.M. and

226 C.C contributed to the behavioral experiments and immunochemistry; S.D., A.E., R. A., H.H.L., A.

227 E., O.D-B and N.H. analyzed the data; H.M.C. S. P. and O.R. critically reviewed the manuscript.

228 S.D., A.E., O.D-B and N.H. wrote the paper.

\section{Acknowledgments}

230 This research was supported by "La Région Rhône-Alpes- SCUSI 2018-\#R18119CC" and

231 "IINSERM" and the ANR Grant "progenID". Whole transcriptome profiling was supported by NIH 232 grant EY 016807.

\section{Competing interests}

234 The author declare no conflict of interests.

\section{References}

236 Cui Y, Yang Y, Ni Z, Dong Y, Cai G, Foncelle A et al. Astroglial Kir4.1 in the lateral habenula drives

237 neuronal bursts in depression. Nature. 2018; 554(7692): 323-327. doi:10.1038/nature25752

238 Defrancesco M, Niederstätter H, Parson W, Kemmler G, Hinterhuber H, Marksteiner J, 239 Deisenhammer EA Bright .ambient light conditions reduce the effect of tryptophan depletion in 240 healthy females. Psychiatry Research. 2013; 210(1): 109-14. doi: $241 \quad$ 10.1016/j.psychres.2013.02.008

242 Delcourte S, Dkhissi-Benyahya O, Cooper HM, \& Haddjeri N. Stress Models of Depression: A 243 Question of Bad Timing. ENeuro. 2017;4(2): 1-2. doi: 10.1523/ENEURO.0045-17.2017

244 Falcon E, Maier K, Robinson SA, Hill-Smith TE, Lucki I. Effects of buprenorphine on behavioral 245 tests for antidepressant and anxiolytic drugs in mice. Psychopharmacology (Berl). 246 2015;232(5):907-915. doi: 10.1007/s00213-014-3723-y 
247 Fernandez DC, Fogerson PM, Lazzerini Ospri L, Thomsen MB, Layne RM, Severin D et al. Light

248 Affects Mood and Learning through Distinct Retina-Brain Pathways. Cell 2018; 175(1): 1-14. doi:

$249 \quad$ 10.1016/j.cell.2018.08.004

250 Gerhard DM, Pothula S, Liu RJ, Wu M, Li XY, Girgenti MJ et al. GABA interneurons are the cellular

251 trigger for ketamine's rapid antidepressant actions. J Clin Invest. 2020; 130(3):1336-1349 doi:

$252 \quad 10.1172 / \mathrm{JCl} 130808$

253 Gigliucci V, O'Dowd G, Casey S, Egan D, Gibney S, Harkin A. Ketamine elicits sustained

254 antidepressant-like activity via a serotonin-dependent mechanism. Psychopharmacology. 2013;

255 228(1): 157-166. doi: 10.1007/s00213-013-3024-x

256 Gonzalez MMC., \& Aston-Jones G. Light deprivation damages monoamine neurons and produces

257 a depressive behavioral phenotype in rats. Proceedings of the National Academy of Sciences of

258 the United States of America. 2008; 105(12): 4898-4903. doi: 10.1073/pnas.0703615105

259 Hattar S, Kumar M, Park A, \& Tong P. Central Projections of Melanopsin-Expressing Retinal

260 Ganglion Cells in the Mouse. Journal of Comparative Neurology. 2006; 497(3): 326-349. doi:

$261 \quad 10.1002 / c n e .20970$

262 Huang L, Xi Y, Peng Y, Yang Y, Huang X, Fu Y et al. A Visual Circuit Related to Habenula Underlies

263 the Antidepressive Effects of Light Therapy. 2019; Neuron 102(1):128-142.e8. doi:

$264 \quad$ 10.1016/j.neuron.2019.01.037

265 Klein ME, Chandra J, Sheriff S, Malinow R. Opioid system is necessary but not sufficient for

266 antidepressive actions of ketamine in rodents. Proc Natl Acad Sci U S A. 2020;117(5):2656-2662.

267 doi: $10.1073 /$ pnas. 1916570117

268 Krishnan V \& Nestler EJ. The molecular neurobiology of depression. Nature. 2008; 455(7215):

269 894-902.

270 Lam RW, Levitt AJ, Levitan RD, Michalak EE, Cheung AH, Morehouse R et al. Efficacy of Bright

271 Light Treatment, Fluoxetine, and the Combination in Patients With Nonseasonal Major

272 Depressive Disorder. JAMA Psychiatry. 2016; 73(1): 56-63. doi: 10.1038/nature07455 
273 Lecca S, Pelosi A, Tchenio A, Moutkine I, Lujan R, Hervé D, Mameli M. Rescue of GABAB and

274 GIRK function in the lateral habenula by protein phosphatase 2A inhibition ameliorates

275 depression-like phenotypes in mice. Nature Medicine. 2016; 22: 254-261. doi: 10.1038/nm.4037

276 LeGates TA, Fernandez DC, \& Hattar S. Light as a central modulator of circadian rhythms, sleep

277 and affect. Nat Rev Neurosci. 2014;18(9): 1199-1216. doi: 10.1038/nrn3743

278 McClung CA. How Might Circadian Rhythms Control Mood? Let Me Count the Ways... Biological

279 Psychiatry. 2013; 74(4): 242-249. doi: 10.1016/j.biopsych.2013.02.019

280 Metzger M, Bueno D, and Lima LB. The lateral habenula and the serotonergic system.

281 Pharmacology Biochemistry and Behavior. 2017; 162: 22-28. doi: 10.1016/j.pbb.2017.05.007

282 Pałucha-Poniewiera A, Podkowa K, Lenda T, \& Pilc A. The involvement of monoaminergic

283 neurotransmission in the antidepressant-like action of scopolamine in the tail suspension test.

284 Progress in Neuro-Psychopharmacology and Biological Psychiatry. 2017; 79: 155-161. doi:

$285 \quad$ 10.1016/j.pnpbp.2017.06.022

286 Pasquier DA, Anderson C, Forbes WB, \& Morgane PJ. Horseradish peroxidase tracing of the lateral 287 habenular-midbrain raphe nuclei connections in the rat. Brain Research Bulletin. 1976; 1(5): 443288 451. doi: 10.1016/0361-9230(76)90114-3

289 Petryshen TL, Lewis MC, Dennehy KA., Garza JC., \& Fava M. Antidepressant-like effect of low 290 dose ketamine and scopolamine co-treatment in mice. Neuroscience Letters. 2016; 620: 70-73. 291 doi: 10.1016/j.neulet.2016.03.051

292 Porsolt RD, Le Pichon M, Jalfre M. Depression: a new animal model sensitive to antidepressant 293 treatments. Nature. 1977;21 266(5604):730-2. doi: 10.1038/266730a0

294 Sun P, Zhang Q, Zhang Y, Wang F, Chen R, Yamamoto R, Kato N. Homer1a-dependent recovery 295 from depression-like behavior by photic stimulation in mice. Physiol Behav. 2015; 147:334-41. 296 doi: 10.1016/j.physbeh.2015.05.007

297 Williams NR, Heifets BD, Bentzley BS, et al. Attenuation of antidepressant and antisuicidal effects 298 of ketamine by opioid receptor antagonism. Mol Psychiatry. 2019;24(12):1779-1786. doi: $299 \quad 10.1038 / \mathrm{s} 41380-019-0503-4$ 
300 Wirz-Justice A, Terman M, Oren DA, Goodwin FK, Kripke DF, Whybrow PC et al. Brightening

301 Depression. Science. 2004; 303: 467-468. doi: 10.1126/science.303.5657.467c

302 Yang Y, Cui Y, Sang K, Dong Y, Ni Z, Ma S, Hu H. Ketamine blocks bursting in the lateral habenula

303 to rapidly relieve depression. Nature. 2018 ; 554(7692): 317-322. doi: 10.1038/nature25509

Table I: Statistical analysis

Figure 1

\begin{tabular}{|c|c|c|}
\hline \multicolumn{2}{|c|}{ ONE WAY REPEATED ANOVA } & Days \\
\hline \multirow{2}{*}{$1 \mathrm{~A}$} & Immobility 5d-RFSS & $\begin{array}{c}\mathrm{F}_{7,84}=8,381 \\
\mathrm{p}<0,0001\end{array}$ \\
\hline
\end{tabular}

\begin{tabular}{|c|c|c|}
\hline \multicolumn{2}{|c|}{ Student T Test } & \\
\hline 1B & Sucrose preference test & $p=0,0091$ \\
\hline
\end{tabular}

\begin{tabular}{|c|c|c|}
\hline \multicolumn{2}{|c|}{ Student T Test } & \\
\hline $1 C$ & Elevated plus maze & $\mathrm{P}=0.0183$ \\
\hline
\end{tabular}

\begin{tabular}{|c|c|c|}
\hline \multicolumn{2}{|c|}{ Student T Test } & Treatment \\
\hline 1D & Latency to feed NSFT (5d-RFSS) & $\begin{array}{c}\mathrm{F}_{2,27}=9,740 \\
\mathrm{p}=0,0007\end{array}$ \\
\hline
\end{tabular}

\begin{tabular}{|c|c|c|}
\hline \multicolumn{2}{|r|}{ ONE WAY REPEATED ANOVA } & Days \\
\hline $1 E$ & Immobility 5d-RFSS + escitalopram & $\begin{array}{c}F_{4,20}=12.710 \\
p=0,0001\end{array}$ \\
\hline
\end{tabular}

\begin{tabular}{|c|c|c|}
\hline \multicolumn{2}{|c|}{ Student T Test } & Treatment \\
\hline 1F & Immobility naïve escitalopram & $\begin{array}{c}\mathrm{t}_{14}=2,349 \\
\mathrm{p}=0,034\end{array}$ \\
\hline
\end{tabular}

\begin{tabular}{|c|c|c|}
\hline \multicolumn{2}{|c|}{ ONE WAY REPEATED ANOVA } & Days \\
\hline \multirow{2}{*}{$1 \mathrm{G}$} & Immobility 5d-RFSS + ketamine & $\begin{array}{c}\mathrm{F}_{6,20}=7.695 \\
\mathrm{p}<0,0004\end{array}$ \\
\hline
\end{tabular}

\begin{tabular}{|c|l|l|}
\hline \multicolumn{2}{|c|}{ Student T Test } & Treatment \\
\hline $1 \mathrm{H}$ & Immobility naïve ketamine & $\begin{array}{l}\mathrm{t}_{16}=2.799 \\
\mathrm{p}=0,0095\end{array}$ \\
\hline
\end{tabular}


Figure 2

\begin{tabular}{|c|c|c|}
\hline \multicolumn{2}{|c|}{ ONE WAY REPEATED ANOVA } & Days \\
\hline $2 A$ & Immobility 5d-RFSS + BLS & $\begin{array}{c}F_{5,30}=10,609 \\
p<0,0001\end{array}$ \\
\hline
\end{tabular}

\begin{tabular}{|c|l|l|}
\hline \multicolumn{2}{|c|}{ Student T Test } & Treatment \\
\hline 2B & $\begin{array}{c}\text { Immobility naïve ketamine+ } \\
\text { scopolamine }\end{array}$ & $\begin{array}{l}\mathrm{t}_{13}=4,663 \\
\mathrm{p}=0,0004\end{array}$ \\
\hline
\end{tabular}

\begin{tabular}{|c|c|c|c|c|}
\hline \multicolumn{2}{|c|}{ TWO WAY REPEATED ANOVA } & Treatment & Days & Interaction \\
\hline $2 C$ & Immobility 5d-RFSS $\mathrm{K}+\mathrm{S}+\mathrm{BLS}$ & $\begin{array}{c}F_{1,10}=0,9228 \\
p=0,8101\end{array}$ & $\begin{array}{c}F_{5,50}=24,096 \\
p<0,0001\end{array}$ & $\begin{array}{c}F_{5,50}=3,329 \\
p=0,0113\end{array}$ \\
\hline \multicolumn{2}{|r|}{ Student T Test } & Treatment & & \\
\hline $2 C$ & Immobility 5d-RFSS K + S+ BLS & $\begin{array}{c}t_{10}=-2,721 \\
p=0,0215\end{array}$ & & \\
\hline
\end{tabular}

\begin{tabular}{|c|c|c|c|c|}
\hline \multicolumn{2}{|c|}{ TWO WAY REPEATED ANOVA } & Treatment & Days & Interaction \\
\hline $2 \mathrm{D}$ & Immobility 5d-RFSS PCPA & $\begin{array}{c}F_{1,15}=0,423 \\
p=0,521\end{array}$ & $\begin{array}{c}F_{5,75}=24,797 \\
p<0,0001\end{array}$ & $\begin{array}{c}F_{5,75}=1,875 \\
p=0,1087\end{array}$ \\
\hline \multicolumn{2}{|r|}{ Student T Test } & Treatment & & \\
\hline $2 \mathrm{D}$ & Immobility 5d-RFSS PCPA & $\begin{array}{l}t_{15}=2,751 \\
p=0,0148\end{array}$ & & \\
\hline
\end{tabular}

\begin{tabular}{|c|c|c|c|c|}
\hline \multicolumn{2}{|c|}{ TWO WAY REPEATED ANOVA } & Treatment & Days & Interaction \\
\hline \multirow{2}{*}{$2 \mathrm{E}$} & Immobility 5d-RFSS DREADD & $\begin{array}{c}\mathrm{F}_{1,14}=10,335 \\
\mathrm{p}=0,0061\end{array}$ & $\begin{array}{c}\mathrm{F}_{5,70}=49,957 \\
\mathrm{p}<0,0001\end{array}$ & $\begin{array}{c}\mathrm{F}_{5,70}=1,598 \\
\mathrm{p}=0,1720\end{array}$ \\
\hline \multicolumn{2}{|c|}{ Student T Test } & Treatment & \multicolumn{2}{|c}{} \\
\cline { 1 - 3 } $2 \mathrm{E}$ & Immobility 5d-RFSS DREADD & $\begin{array}{c}\mathrm{t}_{14}=2,377 \\
\mathrm{p}=0,0323\end{array}$ & \\
\end{tabular}


A
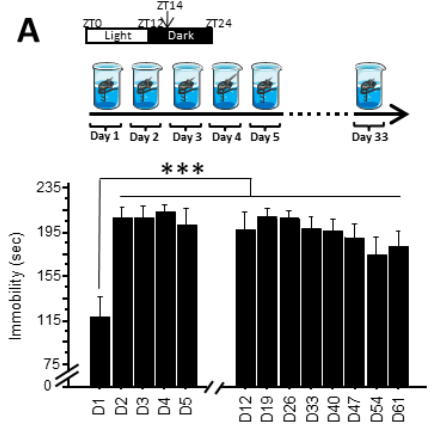

$\mathbf{E}$

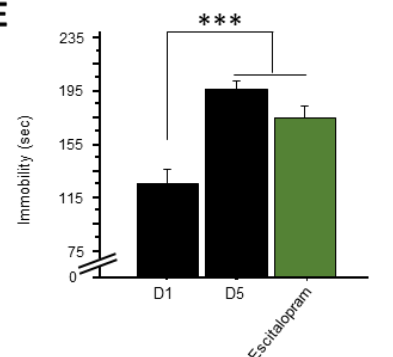

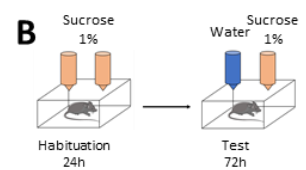

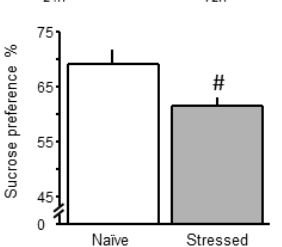

$\mathbf{F}$
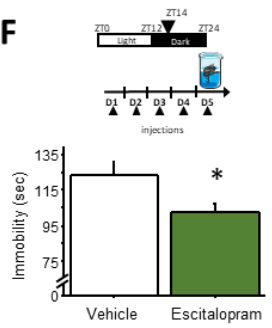

C
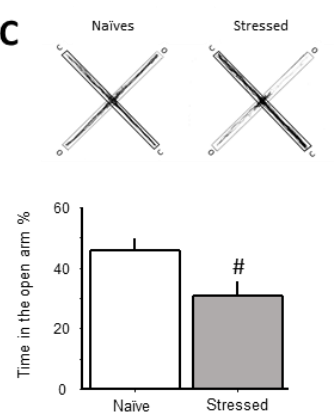

G

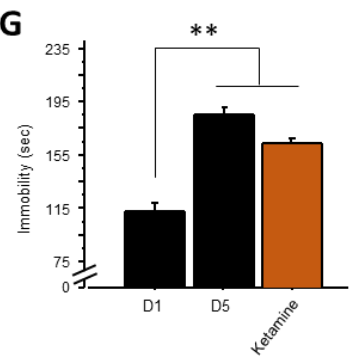

D
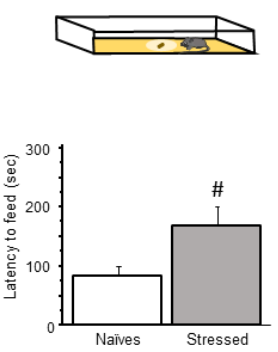

H

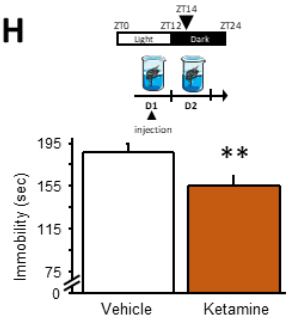

Figure 1. 5d-RFSS: a resistant model of depression

(A) 5d-RFSS: 6 weeks-old C57BL/6J mice were forced to swim for 5 successive days [D] and for 10 minutes at ZT14. Animals were then tested every week $(n=8)$, ${ }^{* *} p<0.0001$ vs D1. (B) Sucrose Preference Test, $48 \mathrm{~h}$ post stress $(\mathrm{n}=8), \# \# \mathrm{p}<0.001$. (C) Elevated plus maze, 1 week post-stress $(n=8-13)$. C =closed arm, $O=o p e n$ arm, \# $p<0.05$. (D) Novelty suppressed feeding test $(n=9-12)$, \# $\mathrm{p}<0.05$. (E) Effect of a sub-chronic escitalopram treatment $(10 \mathrm{mg} / \mathrm{kg}$ i.p) 5 consecutive days from D10 to D15 treatment, $(n=8) p<0.0001$ vs D1. (F) Effect of a sub chronic escitalopram treatment on naïve mice $(n=8){ }^{*} p<0.05(\mathbf{G})$ Effect of acute ketamine treatment $(10 \mathrm{mg} / \mathrm{kg}$, i. $\mathrm{p}), \mathrm{n}=6,{ }^{* *} \mathrm{p}<0.001 \mathrm{vs}$ D1. (H) Effect of acute ketamine treatment on naïve mice $(10 \mathrm{mg} / \mathrm{kg}$, i.p) unpaired Student's t-tests; ${ }^{* *} \mathrm{p}<0.01(\mathrm{n}=13-15)$. Data are expressed as means \pm S.E.M. 
A
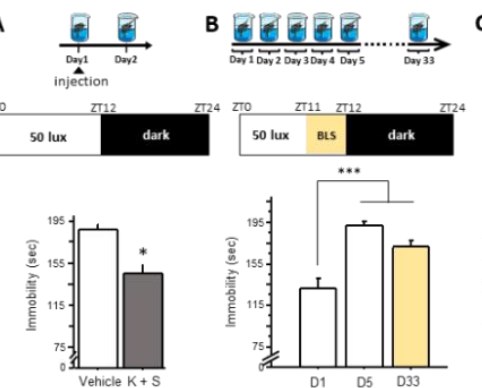

F
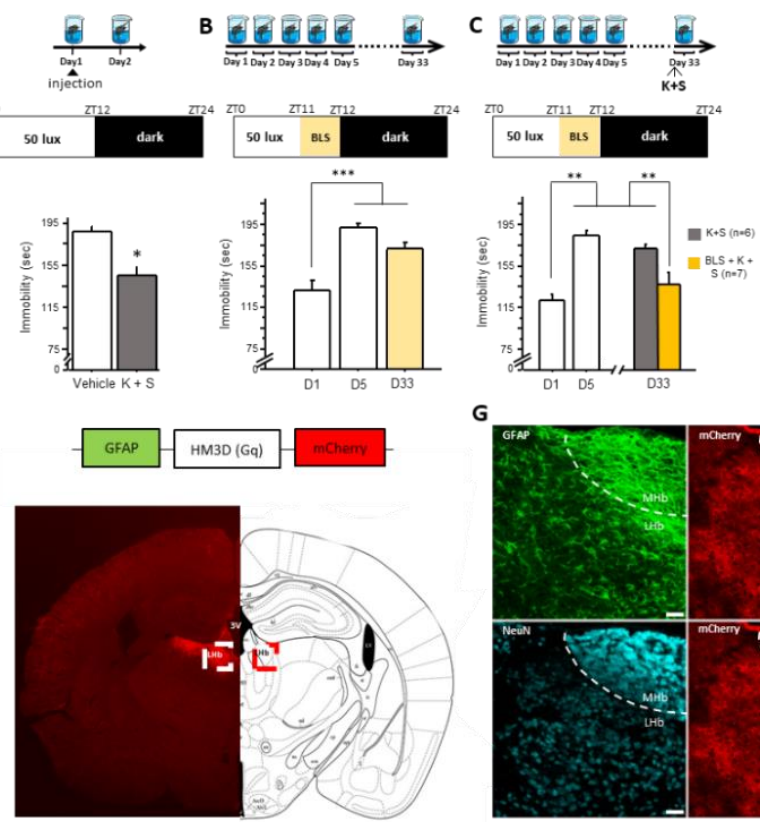

G

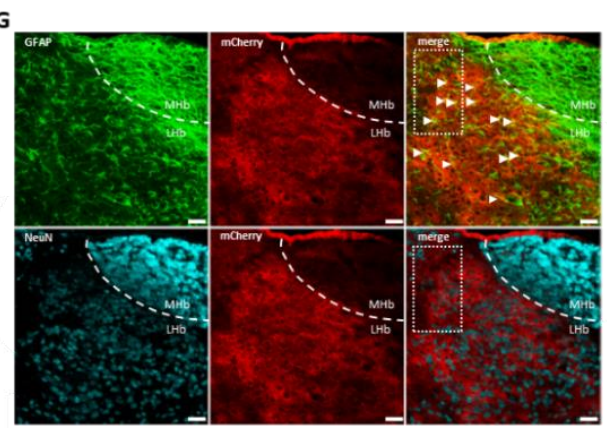

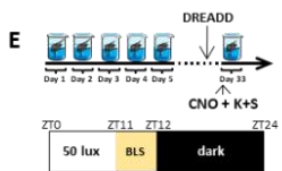
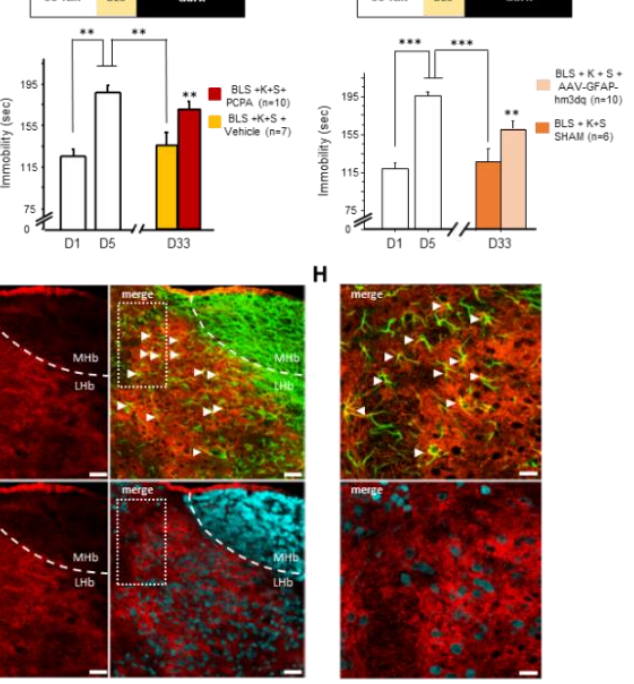

Figure 2. BLS potentiates the AD response of a combination of ketamine and scopolamine.

(A) Effect of a pharmacological co-treatment with Ketamine [K] (3mg/kg, i.p.) and Scopolamine [S] $\left(0.1 \mathrm{mg} / \mathrm{kg}\right.$, i.p.) in naïve mice $\left({ }^{*} P<0.05, n=8\right)$. (B) Effect of a $B L S$ on the increase of immobility time in $5 d$-RFSS model $(n=6),{ }^{* *} P<0.0001$ vs D1. (C) Effect of a combination of BLS and pharmacological co-treatment with $K+S(n=6-7) .{ }^{* *} P<0.01$ vs $D 1$ or D33. On day 33, the two groups were compared. (D) Involvement of 5-HT neurotransmission: mice received a dose of $150 \mathrm{mg} / \mathrm{kg} /$ day (i.p) of PCPA to reduce the levels of $5-\mathrm{HT}(\mathrm{n}=7-10)$. ${ }^{* *} \mathrm{P}<0.01 \mathrm{vs} \mathrm{D1}$ or D33. On day 33, the two groups were compared. (E) Implication of $L H b$ astrocytes in the AD effect of BLS: Following 5d-RFSS, mice were intracerebrally injected with vehicle or a AAVGFAP-Gq-m-cherry virus in the LHb. A 4-week BLS was then applied. At D33, mice received an injection of Clozapine-n-oxide [CNO] (1 mg/kg, i.p.) 1 hour before the FST. Half an hour after the CNO injection, co-treatment $[\mathrm{K}+\mathrm{S}]$ (respectively 3 and $0.1 \mathrm{mg} / \mathrm{kg}$ i.p) was administered to the mice $(n=6-10) .{ }^{* * *} \mathrm{P}<0.01$ vs $\mathrm{D} 1$ or $\mathrm{D} 33$. On day 33 , the two groups were compared. $(F)$ Localization of AAV-GFAP-Gq-m-cherry virus in the $L H b$ : Coronal image of brain section showing mCherry labelling (red) in the LHb (white square). $(\mathbf{G}, \mathbf{H})$ Overview of astroglial and neuronal staining in the LHb: Anti-GFAP (green) and anti-NeuN (blue). Scale bars $=150 \mu \mathrm{m}(\mathrm{G})$, $75 \mu \mathrm{m}(\mathrm{H})$. Datas are expressed as means \pm S.E.M. 